\title{
Determination of heat transfer into a wedge model in a hypersonic flow using temperature-sensitive paint
}

\author{
Steffen Risius · Walter H. Beck · Christian Klein · Ulrich Henne · Alexander \\ Wagner
}

\begin{abstract}
Heat loads on spacecraft travelling at hypersonic speed are of major interest for their designers. Several tests using temperature-sensitive paints (TSP) have been carried out in long duration shock tunnels to determine these heat loads; generally paint layers were thin, so that certain assumptions could be invoked to enable a good estimate of the thermal parameter $\rho c k$ (a material property) to be obtained - the value of this parameter is needed to determine heat loads from the TSP. Very few measurements have been carried out in impulse facilities (viz. shock tunnels such as the High Enthalpy Shock Tunnel (HEG)), where test times are much shorter. Presented here are TSP temperature measurements and subsequently derived heat loads on a ramp model placed in a hypersonic flow in HEG (specific enthalpy $h_{0} \approx 3.3 \mathrm{MJ} \mathrm{kg}^{-1}$, Mach number $M=7.4$, temperature $T=$ $277 \mathrm{~K}$, density $\rho_{\infty}=11 \mathrm{~g} / \mathrm{m}^{3}$ ). A number of fluorescence intensity images were acquired, from which, with the help of calibration data, temperature field data on the model surface were determined. From these the heat load into the surface was calculated, using an assumption of a $1 \mathrm{D}$, semi-infinite heat transfer model. $\rho c k$ for the paint was determined using an insitu calibration with a Medtherm coaxial thermocouple mounted on the model; Medtherm $\rho c k$ is known. Finally presented are sources of various measurement uncertainties, arising from: (1) estimation of $\rho c k$ (2) intensity measurement in the chosen interrogation area (3) paint time response.
\end{abstract}

Steffen Risius

steffen.risius@dlr.de

Institute of Aerodynamics and Flow Technology, German Aerospace Center (DLR), Bunsenstr. 10, 37073 Göttingen, Germany

First published by Springer-Verlag GmbH Germany 2017

Exp Fluids (2017) 58:117

doi $10.1007 / \mathrm{s} 00348-017-2393-\mathrm{z}$

\section{Introduction}

Test models coated with temperature-sensitive paints (TSP) have been used in the DLR over recent years in various wind tunnels (WT) to measure temperatures and/or temperature changes on the model surface - see Costantini et al. (2015); Engler et al. (2000); Fey et al. (2013); Klein et al. (2005. 2013, 2014); Streit et al. (2011) for results with TSP (and also with its pressure analogue PSP - this is also referenced here since many details and difficulties of the technique are common to both TSP and PSP). Absolute temperatures as a function of time during the flow are needed to determine heat transfer from the (usually much hotter) test gas into the model surface - this will be the main topic of interest in this paper, and will be further elucidated in following sections. (Measurement of temperature changes alone can be used to determine boundary layer transition in some WT flows this will not be further discussed here, but an example can be seen in Costantini et al. (2015).) Since objects (missiles, space transporters and capsules, ...) moving at supersonic and especially hypersonic speeds through some atmospheric gas are exposed to very high gas temperatures behind the generated bow shock, it is essential for the optimization of the external aerodynamics and for the design of the insulation of these vehicles to be able to determine the heat loads that prevail during the various stages of the ascent and descent trajectories. The WT used are often so-called impulse facilities (such as shock tubes or tunnels), giving test times in some cases only in the millisecond (or even microsecond) range; this provides a particular challenge for the TSP technique to be able to carry out measurements in these short times. In recent years, an increased interest (Gardner et al. 2014, Gregory et al. 2014) in the study of instationary flow physical phenomena (transition, turbulence, separation, heat transfer) has led to a requirement for fast acquisition systems: high speed cameras, high power (pulsed) LED's and, above all, paints with sufficiently fast response times to en- 
able these instationary or short-duration phenomena to be captured quantitatively and faithfully.

Some of the first measurements using TSP (and PSP) in a shock tunnel to measure heat loads (and pressure distribution) on a test model were carried out in the early 2000's by the research group of Prof. Asai (then at NAL Chofu, Japan, now at Tohoku University in Sendai, Japan): see, for example Nakakita et al. (2003); Ohmi et al. (2006), wherein other work in hypersonic flows is also referenced. The $0.44 \mathrm{~m} \mathrm{Hy-}$ personic Shock Tunnel (NAL-HST) has two different operation modes to generate the shock: the Long Duration Mode (1) using fast-acting valves or the High Enthalpy Mode (2) using a bursting diaphragm, with both modes delivering flows with different durations of about $20 \mathrm{~ms}$ and $1 \mathrm{~ms}$ to $5 \mathrm{~ms}$, respectively. The TSP measurements cited in the above-mentioned papers were carried out using operation mode (2). The paint consisted of a Ru(phen) luminophore embedded in a polyacrylic acid binder. Very thin layers $(\leq 1 \mu \mathrm{m})$ were applied, and the assumption was then made that the temperature measured by TSP differs only slightly from that under the layer on the model surface - this difference was estimated to be only about $4 \%$. Hence, one could take values for the thermal parameter ( $\rho c k)$ for the model material (known) rather than that of the TSP layer (unknown) to calculate heat load from the measured TSP temperature. The thin TSP layers have the disadvantage, however, that the fluorescence intensity is quite low, although this is to some extent compensated by the long flow test times of around $20 \mathrm{~ms}$. In a recent paper by Peng et al. (2016) the results from PSP and TSP measurements carried out in a long-duration hypersonic tunnel have also been presented.

In this paper a different approach is suggested for measurements in the DLR High Enthalpy Shock Tunnel (HEG) with its much shorter test times $(<10 \mathrm{~ms})$. The DLR Eubased paint OV322 active (see later) used here has a high fluorescence intensity, but is much thicker $(80 \mu \mathrm{m})$ than that used in long duration tunnels, so that the assumptions for $\rho c k$ made there do not apply here. Here a Medtherm coaxial thermocouple (see later) is flush mounted with the coated test surface, so that temperature measurements from both TSP and Medtherm are available; one can use contiguous regions for temperatures from Medtherm and TSP to do an insitu calibration of $\rho c k$ for the TSP layer, using the known value for the Medtherm. Measurement uncertainties and errors are discussed in great detail in the present paper. (Whilst preparing this manuscript, a measurement using TSP in the High Enthalpy Shock Tunnel HIEST had been presented at the recent 55'th AIAA Aerospace Sciences Meeting: $\mathrm{Na}$ gayama et al. (2017).)

Flows in hypersonic facilities present several challenges in adapting the TSP technique to these difficult environments, especially in HEG with its test times of less than $10 \mathrm{~ms}$; however, these challenges have been met and addressed, and are discussed in a recent paper which was presented at the 2015 AIAA SciTech Meeting (Beck et al., 2015). TSP based on a different (blue-shifted) luminophore has been studied by Martinez Schramm et al. (2015) and used successfully in HEG to visualize boundary layer transition (Ozawa et al. 2015) and to examine internal SCRAMJET flows (Laurence et al., 2012).

Presented here are temperature measurements and derived heat loads using TSP on a ramp model placed in a hypersonic flow in HEG in order to demonstrate the feasibility of measuring heat transfer into the model surface under these conditions. Results from one only available HEG run are presented. A discussion of derived $\rho c k$ values for the paint is presented, along with a more detailed analysis of measurement errors arising from uncertainties in $\rho c k$ values, statistical fluctuation in the interrogation area and the finite time response of the paint relative to the camera shutter window.

\section{Experimental section}

The HEG tunnel, the test model (ramp) and TSP measurement procedure have been described in greater detail elsewhere (Hannemann and Martinez Schramm, 2007; Hannemann et al., 2008), so that only a short summary will be presented here. Temperature measurements on a ramp model in a low enthalpy flow in the DLR free piston-driven shock tunnel HEG were carried out at one location with a Medtherm thermocouple and over the whole surface which was coated with the TSP paint. The used HEG test condition XIV (Hannemann et al. 2008) was chosen in order to minimize the effect of HEG background radiation (Beck et al., 2015) with specific enthalpy $h_{0} \approx 3.3 \mathrm{MJ} \mathrm{kg}^{-1}$, Mach number $M=7.4$ and the freestream properties temperature $T_{\infty}=277 \mathrm{~K}$ and density $\rho_{\infty}=11 \mathrm{~g} / \mathrm{m}^{3}$. A schematic drawing of HEG is shown in Fig. 1 .

The ramp model, consisting of a flat aluminium plate (with dimensions $80 \mathrm{~mm} \times 20 \mathrm{~mm}$ ) with a sharp leading edge was placed at an angle of $15^{\circ}$ to the flow direction. It was coated with the DLR OV322 paint (Eu-based luminophore and polyurethane (PU) binder), which had been developed in collaboration with the University of Hohenheim (Ondrus et al. 2015). The ramp model had two small holes, one for a point-wise pressure measurement (using a Kulite ${ }^{\circledR}$ piezoresistive pressure sensor mounted below the plate in the plate holder) and one for a point-wise temperature measurement (using the coaxial thermocouple Medtherm ${ }^{\circledR}$ protruding from the holder and inserted in the ramp model hole so as to be flush-mounted with the plate surface) - see Figs. 2 and 3 .

TSP fluorescence was excited with a Rapp ${ }^{\circledR}$ High Power LED operating at $405 \mathrm{~nm}$ in $\mathrm{CW}$ mode and the images were captured with a Photron ${ }^{\circledR}$ HiSpeed Camera SA1 (provided by LaVision ${ }^{\circledR}$ ) running at a sampling rate of $5 \mathrm{kHz}$ and with 


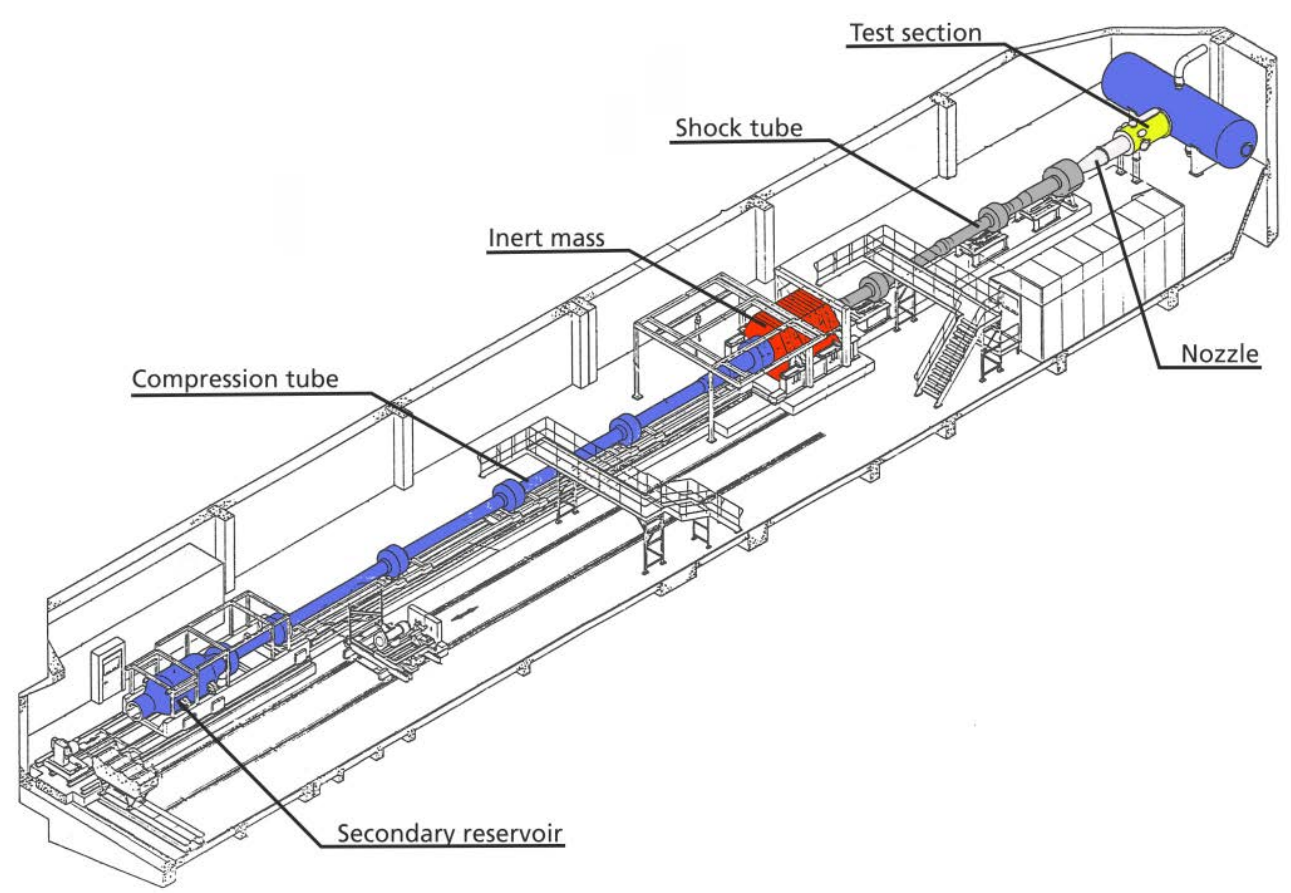

Fig. 1 Schematic drawing of the High Enthalpy Shock Tunnel Göttingen, HEG. Source: Wagner et al. (2013)

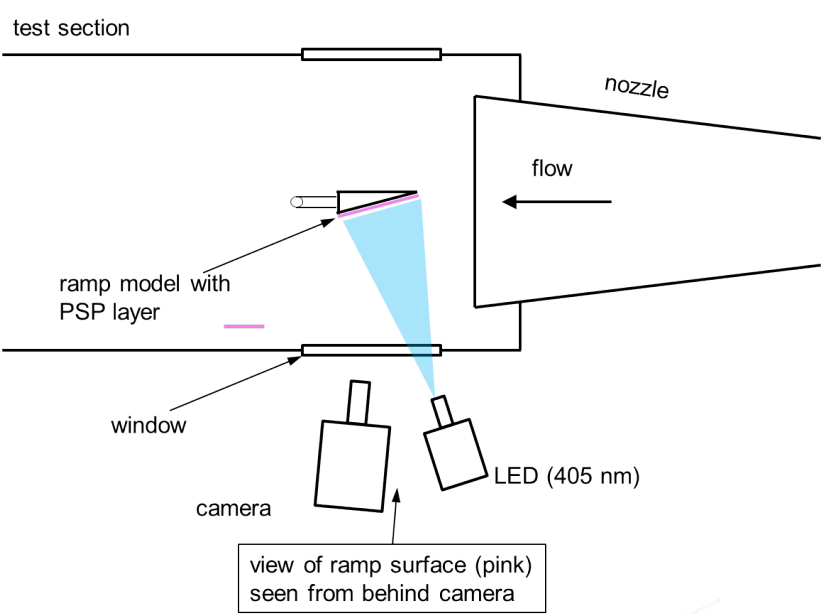

Fig. 2 Block diagram (plan view) of experimental setup in HEG test section.

exposure times of $200 \mu \mathrm{s}$; recordings were carried out over $10 \mathrm{~ms}$, so that 50 images in all were obtained.

\section{Temperature determination using TSP}

The quenching processes which reduce the intensity of the excited paint luminophore fluorescence $I(T)$ are temperature dependent: $I(T)=f(1 / T)$. Typically, using an Arrhe-

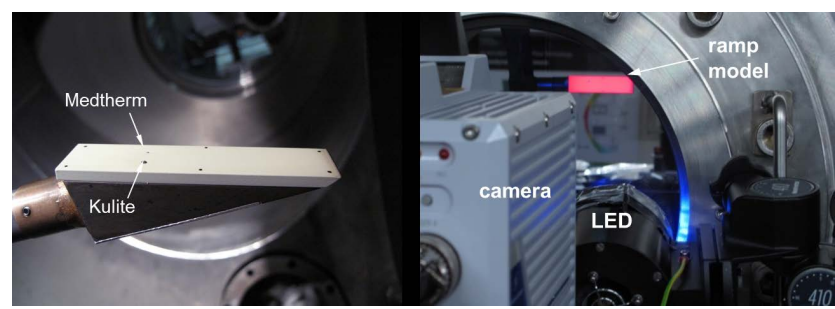

Fig. 3 Left: Detailed photograph of the ramp model, mounted in the test section. (Flow is from right to left.) Right: Photo of irradiated ramp model (pink color) in test section, showing camera and LED light source.

nius formulation, one obtains (Liu and Sullivan, 2005):

$\ln \frac{I(T)}{I\left(T_{r e f}\right)}=\frac{E_{a}}{R}\left[\frac{1}{T}-\frac{1}{T_{r e f}}\right]$

Here $E_{a}$ is the activation energy for the non-radiative process and $R$ is the universal gas constant. In practice, Eq. 1 does not always hold exactly, so that one tends (Liu and Sullivan. 2005) to carry out a calibration at known $T$ (and $P$ ) and perform a fit (e.g. polynomial) of $I(T) / I\left(T_{r e f}\right)$ to $T / T_{\text {ref }}$ :

$\frac{I\left(T_{r e f}\right)}{I(T)}=f\left(T / T_{r e f}\right)$

This is the standard approach adopted here, and was carried out in the laboratory using small coupons coated with the same paint as used on the test model and then calibrated in a test rig with known test conditions (viz. temperature $T$ and pressure $p$ ). A calibration plot for the OV322 paint used here 
is shown in Fig. 4, from which can be seen that this paint has good temperature sensitivity in the range $290 \mathrm{~K}$ to $340 \mathrm{~K}$ (ca. $-3 \% \mathrm{~K}^{-1}$ to $-5 \% \mathrm{~K}^{-1}$ (Ondrus et al. 2015) 1

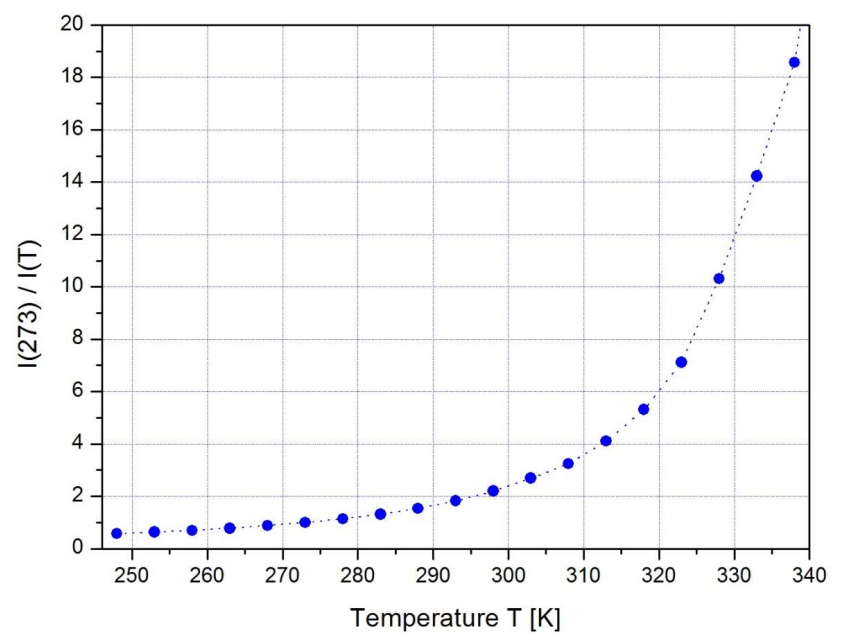

Fig. 4 Calibration of OV322 paint: intensity ratio $I(273 K) / I(T)$ vs. temperature $T$. (Source: Ondrus et al. (2015).

With this calibration plot, and assuming that the conditions used in the HEG test were sufficiently similar (camera settings, lens settings, LED light source) to those of the calibration, one needs only one reference image in HEG to obtain the temperature results for all other images; these reference images in HEG are those which are obtained before gas arrival (at $t<1 \mathrm{~ms}$ ) - see Fig. 5 and later discussion in Sects. 5.1 and 5.2 .

\section{Determination of heat loads}

\subsection{Temperature sensors}

Measurement of heat transfer into a model surface for hypersonic flows of high enthalpy (up to $22 \mathrm{MJ} \mathrm{kg}^{-1}$ ) and short duration (down to $100 \mu \mathrm{s}$ ) places stringent requirements on the performance of the adopted temperature sensors: they must have a fast response and be able to survive the extreme environments of these flows (especially the high temperatures and, in some cases, presence of foreign particulate matter). Over the years coaxial thermoelements have become established as the sensor of choice in HEG (and most shock tunnels) to fulfil the abovementioned requirements. The firm Medtherm supplies these sensors, and also provides a calibration (see later) to be used for the evaluation of heat transfer from temperature measurements. Obviously, these sensors yield point-wise measurements only, so that

\footnotetext{
${ }^{1}$ The normalization with a different temperature would also be possible, which would lead to a constant multiplicative factor. See for example Ondrus et al. (2015).
}

a test model must be equipped with many to obtain overall heat loads; this may be difficult, especially with some complex geometries. The advantage of a field measurement technique such as TSP lies clearly to hand.

\subsection{Evaluation of heat transfer from Medtherms and TSP}

Following an approach suggested by Schultz and Jones (1973), with a modification by Cook and Felderman (1966), two key assumptions were made to obtain a simple relationship for determining heat transfer from temperature measurements:

1. Heat transfer into the model surface (or paint) is onedimensional;

2. The surface is assumed to be semi-infinite in depth.

Obviously these requirements are only met if the time over which measurement occurs is very short - for the typical test times in shock tunnels $(<10 \mathrm{~ms})$, it can be shown (Martinez Schramm et al. 2015) that these assumptions are valid. Following Hannemann and Martinez Schramm (2007) (wherein a good discussion of heat transfer measurements in shock tunnels can be found), the following equation for heat transfer has been derived:

$q_{w}(t)=\sqrt{\frac{\rho c k}{\pi}}\left[\frac{T(t)}{\sqrt{t}}+\frac{1}{2} \int_{0}^{t} \frac{T(t)-T(\tau)}{(t-\tau)^{3 / 2}} d \tau\right]$

where $T=$ measured temperature, $\rho=$ material density; $c=$ heat capacity; $k=$ heat conductivity; $\tau=$ time; $t=$ integration time step. The factor $\rho c k$ is also known as the thermal parameter, and is strictly a function of the (sensor) material properties only. This is not fully correct, since $c$ and $k$ may also have temperature dependence - see discussion in Sect. 5.4. Since there are no available calibration values for $\rho c k$ of the TSP paint (but see later), the approach adopted here is the following: using temperatures measured by the Medtherm sensor, and using its known $\rho c k$ value (as supplied by the manufacturer: $\rho c k=7.95 \times 10^{7} \mathrm{~kg}^{2} \mathrm{~K}^{-2} \mathrm{~s}^{-5}$ ), determine the heat load at its position using a discretized form of Eq. 3. Then assume that the heat load in an adjoining interrogation region (see Fig. 5, to be discussed later) is the same as for the Medtherm location, so that, by adjusting (varying) the $\rho c k$ value for the TSP paint in this region, the same heat load as for the Medtherm is determined. This is effectively an insitu calibration of the TSP paint, with the found $\rho c k$ being assumed to be the same and applicable over all the whole coated surface.

\section{Results and discussion}

This section addresses the following: raw TSP results; TSP temperature results; TSP heat load results; an estimation of 
the accuracy of and uncertainties in the TSP temperature and heat load results.

\subsection{HEG results; TSP images (raw data)}

The results from only one HEG run were available, so that measurements of reproducibility could not be carried out. The temperature (Medtherm) and adjacent pressure (Kulite) measurements at the respective sensor locations (as shown in the inset in Fig. 5 are given in Fig. 6 and Fig. 7, temperature is plotted as $\Delta T$ (where $T_{0}=293 \mathrm{~K}$ before gas arrival) as a function of time. The bump seen peaking at $2 \mathrm{~ms}$ in the Medtherm trace (also in the Kulite trace, but less marked) is due to the nozzle starting processes for this HEG condition. Fifty TSP images were recorded, one every $200 \mu$ s, over the $10 \mathrm{~ms}$ test time period. Fig. 5 shows one of these raw images (with only background correction applied, but with no correction by a reference image).

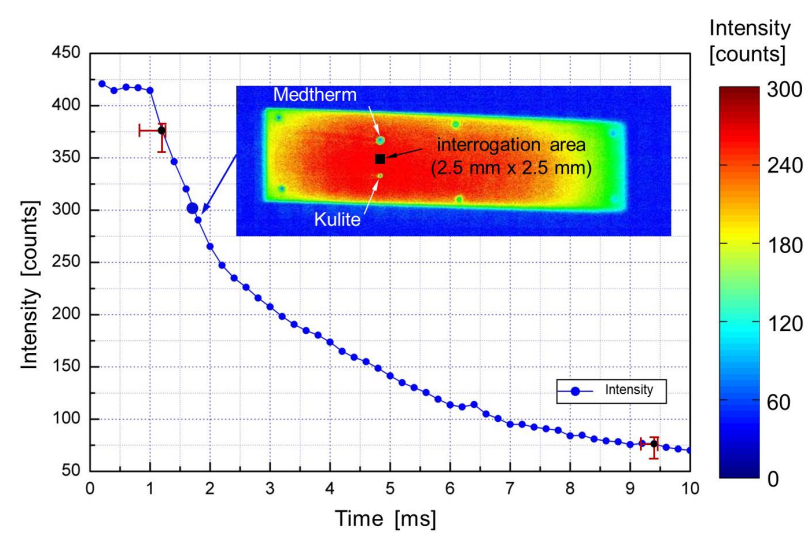

Fig. 5 Average intensity (counts) in interrogation area vs. time (ms) for all 50 recorded raw images. Intensity and time error bars - note asymmetry - for early and late times are shown. A detailed discussion about the measurement uncertainty is given in Sect. 5.4.3 Inset: raw unprocessed image at $t=1.8 \mathrm{~ms}$ showing interrogation area. (Flow in the image is from right to left)

As mentioned before, and for reasons alluded to there, a small (square) interrogation region close to the Medtherm sensor location was chosen; it was situated equidistant between the Medtherm and Kulite sensors, and had a size of $15 \times 15$ pixels, which corresponds to an area on the ramp of approximately $2.5 \mathrm{~mm} \times 2.5 \mathrm{~mm}-$ see inset in Fig. 5 The average (over all pixels) intensity in this region was determined for all 50 images, and then plotted as a function of time, as also shown in Fig. 5. (Intensity and time error bars - note asymmetry - for early and late times are shown: for a discussion, see Sect. 5.4.3.) The abscissa time zero (origin) corresponds to the arrival of the shock wave at the end of the driven section, at which time data recording is triggered. It can be seen that it takes about $1 \mathrm{~ms}$ for the test gas to arrive at the test section window; hence, for times less than $1 \mathrm{~ms}$, the intensity is at a maximum, after which it subsequently drops as the model surface temperature increases.

The results at $t<1 \mathrm{~ms}$ will later be used as reference values, since the conditions (viz. temperature) are well-known at these early times before the test gas has arrived. They will also be used to apply intensity distribution corrections to the results at $t>1 \mathrm{~ms}$, allowing for various geometrical effects (camera angle and settings), and for the uneven LED illumination - see Sect. 4.2 Interestingly, the bump seen clearly in the Medtherm result at $t=2 \mathrm{~ms}$, as referred to before (see Figs. 6and 7), appears as a change of gradient in the TSP result at $t \approx 2.5 \mathrm{~ms}$ - see Fig. 8 . This slight time shift between the Medtherm and TSP results is due to the finite response time of the TSP paint, and will be discussed in more detail in Sect. 5.4.3. The evolution (drop) of intensity is as expected from the model described by Eq. 3 and with the inherent assumptions referred to before. Note from Fig. 5 that the intensities after $7 \mathrm{~ms}$ are only about 100 counts or less, so that the accuracy of the temperature determination will be lower than at early times. This is not a serious restriction, however, since typically the customarily adopted HEG test time window lies much earlier than this (around $3 \mathrm{~ms}$ to $4 \mathrm{~ms}$ ) (see for example Laurence et al. (2014)); the flow at later times may be influenced by effects such as driver gas arrival and presence of other disturbances such as boundary layer leakage, arrival of the contact surface or of the reflected expansion wave, and so is often not further considered.

The change in intensity distribution on the model surface over time, brought about by the increasing surface temperature, can be clearly seen in the four raw images shown in Fig. 8 for the times $t=1.8 \mathrm{~ms}, 2.6 \mathrm{~ms}, 5.0 \mathrm{~ms}$ and $9.0 \mathrm{~ms}$. As expected, the average intensity drops steadily. At this stage, however, without application of the reference image corrections referred to before, nothing can be inferred about the change of temperature as a function of time over the surface - this will be addressed in the next section. Nevertheless, one can already see some physical features: for example, a wake downstream of the Medtherm (its position is shown in Fig. 5), clearly visible at $t=2.6 \mathrm{~ms}$. The wake most likely arises from the sensor being mounted not fully flush with the paint surface, but recessed slightly (by about $10 \mu \mathrm{m}$ to $100 \mu \mathrm{m}$ ) below it ${ }^{2}$ Note again that the results at later times are most likely subject to disturbances which influence the state of the test gas; in spite of this, the development in intensity over time all the way up to $10 \mathrm{~ms}$ looks reasonable and believable.

\footnotetext{
${ }^{2}$ The slight dent at the sensor location is not expected to influence the temperature and heat flux measurements of the Medtherm sensor significantly, since the boundary layer thickness at the sensor location is expected to be at least one order of magnitude larger.
} 




Fig. 6 Temperature change $\Delta T$ measured by a Medtherm sensor on ramp model in this HEG run.

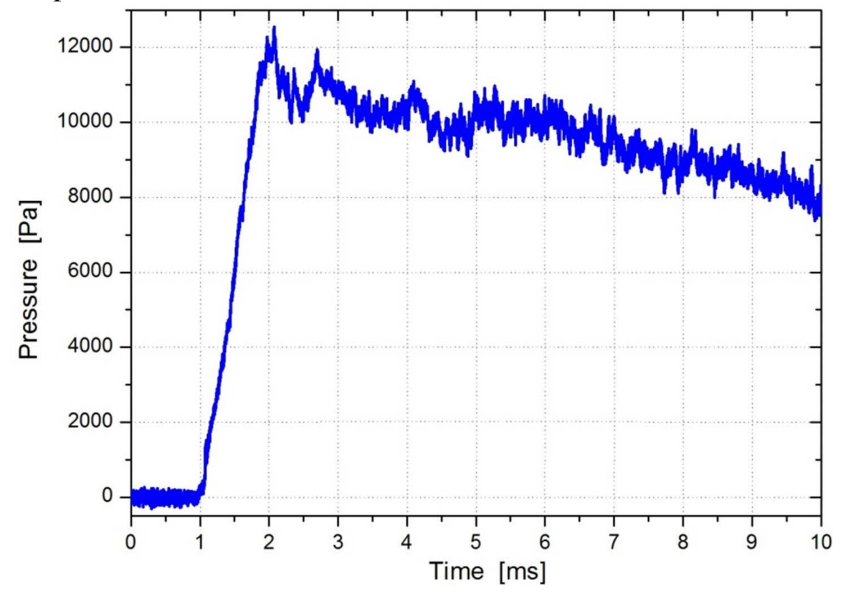

Fig. 7 Pressure change $P$ measured by a Kulite sensor on ramp model in this HEG run.

\subsection{TSP temperature results}

Using the first four recorded images as reference $\left(T_{\text {ref }}=\right.$ $293 \mathrm{~K}$ ), and with the help of the calibration plot shown in Fig. 4. one can then obtain temperatures over the whole model surface for all remaining 46 images. Ten results at different times $(0.4 \mathrm{~ms}, 2 \mathrm{~ms}, 3 \mathrm{~ms}, 4 \mathrm{~ms}, 5 \mathrm{~ms}, 6 \mathrm{~ms}, 7 \mathrm{~ms}$, $8 \mathrm{~ms}, 9 \mathrm{~ms}$ and $10 \mathrm{~ms}$ ) are shown in Fig. 9. where the first image (at $t=0.4 \mathrm{~ms}$ ) is a typical reference image before gas arrival. (The apparent asymmetry (e.g. see direction of the streak wakes) is due to non-parallel alignment of the wedge relative to the flow direction, which can already be inferred from the non-horizontal orientation of the raw images seen in Figs. 5and 8, )

Quite clearly one can see the development of temperature on the surface over time. (The result at $t=0.4 \mathrm{~ms}$ corresponds to a reference image, viz. before gas arrival.) $\mathrm{Re}$ sults for $t>5 \mathrm{~ms}$ suggest temperatures in excess of $330 \mathrm{~K}$ close to the leading edge; these lie outside the calibration range, so that the TSP results close to the leading edge for

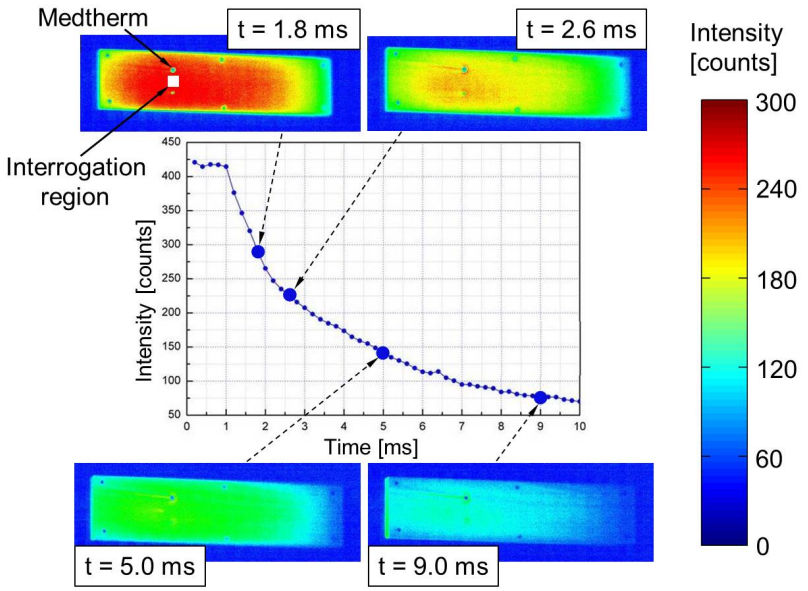

Fig. 8 Average intensity (counts) in interrogation region vs. time (ms) for all 50 recorded raw images, showing four sample raw intensity images at times shown. (Flow in these images is from right to left)

these late times can only be seen as semi-quantitative. However, it should be noted that the used TSP has been shown to work up to $380 \mathrm{~K}$ in a laboratory environment (Ondrus et al. 2015).

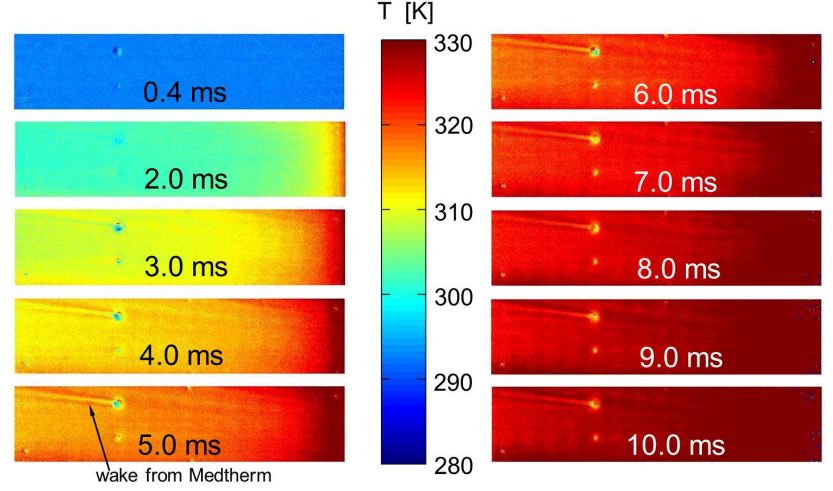

Fig. 9 TSP temperature results (2D images) over the whole model surface for 10 different times during the HEG run. (Flow in these images is from right to left)

One can now plot the obtained average TSP temperatures in the interrogation region (referred to before) for all 46 images and compare the temperature development with the Medtherm results - this comparison is shown in Fig. 10 (Temperature and time error bars for early and late times are shown: for a discussion, see later.) Even though the TSP results after $t=2.5 \mathrm{~ms}$ look good and follow the expected trend, nevertheless recall the remarks made earlier. The difference in development of the actual temperature values obviously arises from the vastly different thermal properties ( $\rho c k$ ) of the Medtherm and TSP paint. This becomes clear from Table 1. where $\rho, c$ and $k$ values (Eng, 2015) for three typical materials are listed, along with the products $\rho c k$ and 
$(\rho c k)^{1 / 2}$ - recall the presence of $(\rho c k)^{1 / 2}$ in Eq. 3. These materials have been chosen as 'representative': Al represents the test model material, Ni the Medtherm and PMMA (polymethylmethacrylate, a polymer commonly used in TSP) the paint. The values of $(\rho c k)$ and $(\rho c k)^{1 / 2}$ for the Medtherm, as supplied by the manufacturer, are also shown. (An aside: note that Ni has different ( $\rho c k$ ) values to that of the Medtherm. This is not surprising, since the Medtherm is not made of just $\mathrm{Ni}$, and some abraded dust from its test preparation has also been rubbed into the sensor.) The final row shows the values of $(\rho c k)^{1 / 2}$ for the various materials normalized to the value $(\rho c k)_{\text {PMMA }}^{1 / 2}$. As can be seen, mainly the much smaller $\mathrm{k}$ (heat conductance) value for PMMA compared with the other three materials leads also to the vastly different $(\rho c k)^{1 / 2}$ values - factors are up to about 40 times larger. The polymer based paint (represented by PMMA in Table 1) cannot conduct away the absorbed heat quickly enough, so that the paint surface heats up to higher values compared to those measured by the Medtherm.

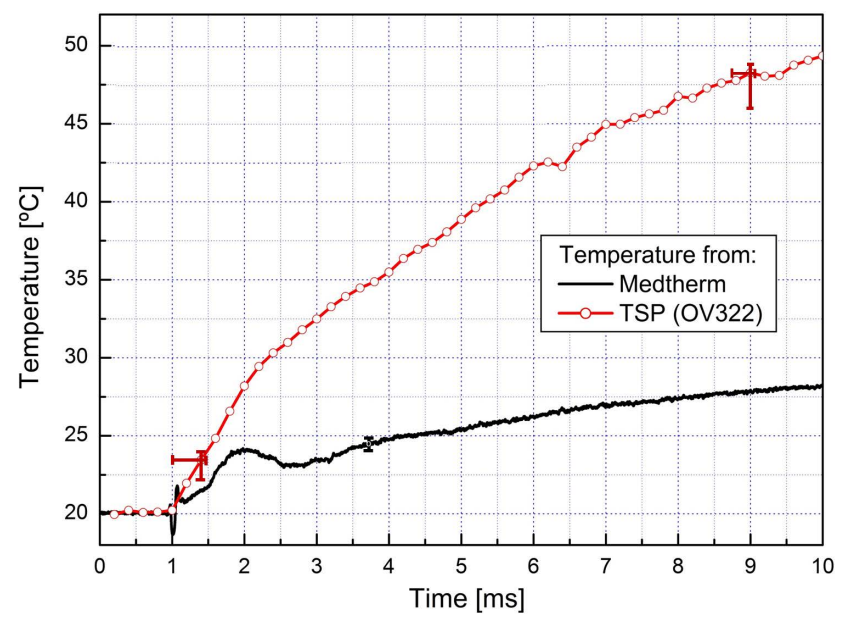

Fig. 10 Medtherm and adjacent TSP temperatures vs. time in the HEG run, the latter using TSP calibration data. (Average TSP temperature in the interrogation area - see Fig.5)

\subsection{TSP heat load results}

Using the discretized form of Eq. 3 referred to before, the TSP temperature results shown in Fig. 9 can now be processed to give the heat loads on the model surface. This local heat load can be determined from the Medtherm measurement at its position (see Fig. 5 for location), so that the TSP average temperatures over time in the adjacent interrogation region can be used to determine a local heat load from TSP measurements, with $\rho c k_{\text {insitu }}$ being varied as a parameter to yield the same heat load from both the TSP and the adjacent Medtherm measurements - this is effectively an insitu calibration of the TSP paint using the Medtherm. The value obtained for the TSP paint with this approach was $\rho c k_{\text {insitu }}=$ $4.5 \times 10^{6} \mathrm{~kg}^{2} \mathrm{~K}^{-2} \mathrm{~s}^{-5}$. It is again assumed that this value of $\rho c k$ pertains over the whole model surface and over the whole time, so that heat loads for all 46 images (from $t=1 \mathrm{~ms}$ to $10 \mathrm{~ms}$ ) over the whole ramp surface can be obtained. (The validity and inherent uncertainties of this assumption will be further discussed in Sect. 5.4) Ten sample heat load results at different times $(0.4 \mathrm{~ms}, 2 \mathrm{~ms}, 3 \mathrm{~ms}, 4 \mathrm{~ms}, 5 \mathrm{~ms}, 6 \mathrm{~ms}$, $7 \mathrm{~ms}, 8 \mathrm{~ms}, 9 \mathrm{~ms}$ and $10 \mathrm{~ms}$ ), as already shown for temperatures in Fig. 9, are shown in Fig. 11 (For the image before gas arrival at $t=0.4 \mathrm{~ms}$ the heat load is obviously zero.) Note that maximum heat loads of $1.6 \mathrm{MWm}^{-2}$ and more are obtained in the upstream (right hand side) positions on the model, even at earlier times (but recall earlier comments on paint calibration and possible damage).


Fig. 11 Heat loads from TSP measurement (2D images) over the whole model surface for 10 different times during the HEG run. (Flow in these images is from right to left)

As before with temperature (Fig. 10, one can now compare the heat load obtained from Medtherm with that from TSP in its adjacent interrogation region. This comparison is shown in Fig. 12 (Average error bars in time and heat transfer are shown: see discussion later.)

Here, at times after $t \approx 3 \mathrm{~ms}$ (i.e. after the initial disturbances seen in the Medtherm results), both heat load results are the same over all times and have average values of $0.73 \pm 0.07 \mathrm{MWm}^{-2}$ for TSP and $0.70 \pm 0.08 \mathrm{MWm}^{-2}$ for Medtherm. But recall: this must obviously be so, since the Medtherm results had been used to calibrate the TSP, so that the agreement has been 'forced'. Nevertheless, the constancy and agreement of heat loads over the whole time for both TSP and Medtherm is very encouraging. The bump in the Medtherm result at $t=1.6 \mathrm{~ms}$ to $2.0 \mathrm{~ms}$ correlates well with the smaller one in the TSP result at $t=2 \mathrm{~ms}$ when one considers the TSP negative time correction of $0.4 \mathrm{~ms}$ due to the finite paint response time - this will be discussed later in Sect. 5.4. 
Table 1 Comparison of material properties ( $\rho$ density, $c$ heat capacity, $k$ heat conductance) and the thermal parameter $(\rho c k)^{1 / 2}$ for nickel Ni (representing the Medtherm thermoelement), aluminum Al (test model) and PMMA (a typical TSP paint polymer). (Source: see text)

\begin{tabular}{llllll}
\hline Property & Units & Medtherm & $\mathrm{Ni}$ & $\mathrm{A} 1$ & PMMA \\
\hline Density $\rho$ & $\mathrm{kg} / \mathrm{m}^{3}$ & - & 8800 & 2712 & 950 \\
Heat capacity & $\mathrm{Jkg}^{-1} \mathrm{~K}^{-1}$ & - & 540 & 870 & 1450 \\
Heat conductance & $\mathrm{Js}^{-1} \mathrm{~K}^{-1} \mathrm{~m}^{-1}$ & - & 90 & 110 & 0.18 \\
$\rho c k$ & $\mathrm{~J}^{2} \mathrm{~m}^{-4} \mathrm{~K}^{-2} \mathrm{~s}^{-1}$ & $7.95 \times 10^{7}$ & $4.3 \times 10^{8}$ & $2.6 \times 10^{8}$ & $2.5 \times 10^{5}$ \\
& $\left(\mathrm{~kg}^{2} \mathrm{~K}^{-2} \mathrm{~s}^{-5}\right)$ & & & \\
$\sqrt{\rho c k}$ & $\mathrm{Jm}^{-2} \mathrm{~K}^{-1} \mathrm{~s}^{-1 / 2}$ & 8915 & 20700 & 16000 & 500 \\
$\sqrt{\rho c k} / \sqrt{(\rho c k)_{P M M A}}$ & $\left(\mathrm{kgK}^{-1} \mathrm{~s}^{-5 / 2}\right)$ & & 41 & 32 & 1 \\
\hline
\end{tabular}

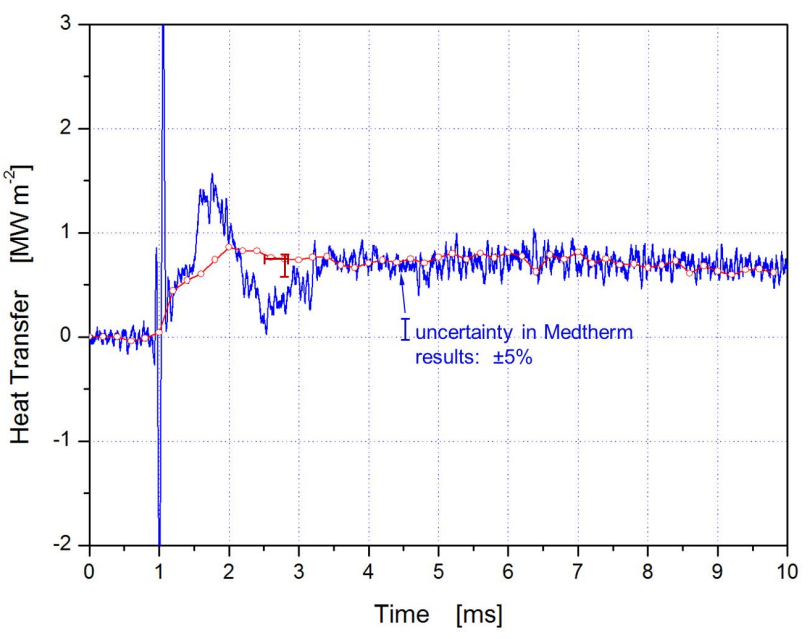

Fig. 12 Comparison of heat load on the ramp from TSP (in the interrogation area) and from Medtherm vs. time. The $\rho c k$ value for TSP was obtained by an insitu calibration using the Medtherm result - see text

\subsection{Accuracy of TSP temperature and heat load results}

Here an attempt will be made to address and identify possible sources of errors leading to uncertainties in the derived temperature and heat load results. These sources are both systematic and random. The final goal will be to determine confidence limits for these measured properties; they have already been shown in the foregoing plots as error bars. Specific other sources such as stray illumination (considered the major problem with quantitative TSP in shock tunnels) and particles (as dust) are not considered explicitly here; they have been discussed elsewhere (Beck et al., 2015). However, for the HEG run condition chosen here (low $h_{0}$, low $\rho_{\infty}$ ), the influence of these sources was minimal and could therefore be neglected here.

\subsubsection{Thermal parameter $\rho c k$ : material properties}

The TSP paint consists of three layers sprayed onto an aluminum substrate: the bottom primer layer, a screen layer and finally the active layer consisting of the polymer (here PU) with its embedded luminophores (Eu complex). Sottong
[DLR Cologne, private communication (2015)] carried out material and thermal analyses of the aluminum substrate and these three layers. Each layer thickness and thence its density were determined; Fig. 13 shows a scanning electron microscope (SEM) image of a sectional cut through the aluminum substrate and its paint coatings, showing the thicknesses of the three layers and the structure of their surfaces. Heat conductance coefficients $k$ for the layers and substrate at temperatures from $25^{\circ} \mathrm{C}$ to $50{ }^{\circ} \mathrm{C}$ were also measured; these values for $k$ (in $\mathrm{Wm}^{-1} \mathrm{~K}^{-1}$ ) are plotted as a function of temperature in Fig. 14 . The coefficient $k$ for aluminum is much larger than that for the active layer, by a factor of about 100. However, in the previous estimate of $k$ in Table 1 , the factor was more like $600(\approx 110 / 0.18)$, which is obviously a huge over-estimate, based on these new measurement results. The conclusion remains the same, however: the main driving force for the different response of the Medtherm and TSP paint to an externally applied heat load is the large difference in $k$. The largest temperature dependence is shown by the innermost primer and the active layers, where k drops by about $15 \%$ from $25^{\circ} \mathrm{C}$ to $50{ }^{\circ} \mathrm{C}$. For the middle screen layer the drop is only about $7 \%$. One can assume (and parametric tests here have shown) that for these short test times $(<10 \mathrm{~ms})$ mainly (but not solely) the material properties of the outer active layer play the more significant role in influencing the heat transfer process.



Fig. 13 SEM (scanning electron microscope) image of cross section through various TSP paint layers, used to determine layer thicknesses (and thence density). Source: see text 


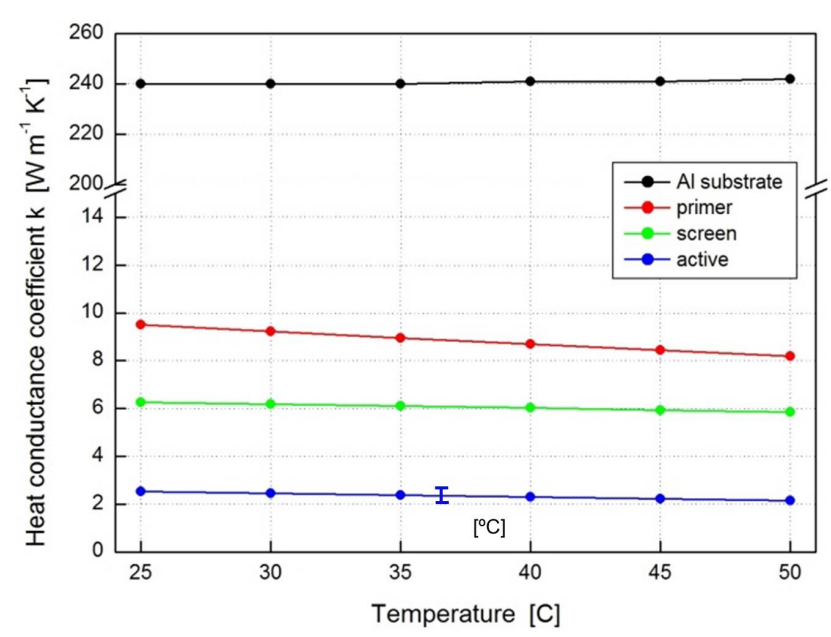

Fig. 14 Measured heat conduction coefficients k as a function of temperature for the three layers making up the TSP paint (error bar for active layer shown). Note break in the ordinate scale. Source: see text

Finally, using the layer properties determined by Sottong, one can obtain a value of $\rho c k$ and compare it with that determined by the Medtherm insitu calibration of the TSP paint (which was $\rho c k_{\text {insitu }}=4.5 \times 10^{6} \mathrm{~kg}^{2} \mathrm{~K}^{-2} \mathrm{~s}^{-5}-$ see before). Recall that this is different than the approach used by (Nakakita et al., 2003), where very thin paint layers were used (see Sect. 11). (For reference, recall that $\rho c k_{\text {Medtherm }}=$ $7.95 \times 10^{7} \mathrm{~kg}^{2} \mathrm{~K}^{-2} \mathrm{~s}^{-5}$ ). Hence, over the temperature range $25^{\circ} \mathrm{C}$ to $50^{\circ} \mathrm{C}$, it is estimated that the assumption of a constant (viz. average) value of $k$ would also lead to an uncertainty (inaccuracy) in the calculated heat loads of about $\pm 5 \%$ (recall: $(\rho c k)^{1 / 2}$, and not $\rho c k$, enters into Eq. 3). Sottong measured a constant value of $c=930 \pm 45 \mathrm{~m}^{2} \mathrm{~s}^{-2} \mathrm{~K}^{-1}$ for all layers and over a temperature range $0{ }^{\circ} \mathrm{C}$ to $50^{\circ} \mathrm{C}$. Taking the TSP sample values supplied by Sottong: $\rho=$ $1330 \mathrm{kgm}^{-3}, \quad c=930 \pm 45 \mathrm{~m}^{2} \mathrm{~s}^{-2} \mathrm{~K}^{-1}$ and $k=2.5 \pm 0.2 \mathrm{Wm}^{-1} \mathrm{~K}^{-1}$ (this value being for just the active layer), one obtains $\rho c k_{\text {mat }}=3.1( \pm 0.3) \times 10^{6} \mathrm{~kg}^{2} \mathrm{~s}^{-5} \mathrm{~K}^{-2}$, based just on the material properties. $\rho c k_{\text {insitu }}$ and $\rho c k_{\text {mat }}$ differ by about $30 \%$. If one assumes that the second screen layer also plays some role in the heat transfer process, this would lead to larger effective values of $k$, lying somewhere in the range $2.3 \mathrm{Wm}^{-1} \mathrm{~K}^{-1}$ (active layer) and $6.1 \mathrm{Wm}^{-1} \mathrm{~K}^{-1}$ (screen), with the subsequent $\rho c k_{\text {mat }}$ then lying between 3.1 and $8.2 \mathrm{~kg}^{2} \mathrm{~s}^{-5} \mathrm{~K}^{-2} . \rho c k_{\text {insitu }}$ is right in the middle of this range. $c$ was measured to be temperature-independent in the range $25^{\circ} \mathrm{C}$ to $50^{\circ} \mathrm{C}$. However, $c$ was measured for the whole sample, and not for the individual layers, which makes it difficult to assess how this simplification may affect the appropriate value of $c$ for the active layer. In conclusion, then, given these uncertainties in the material properties, and based on present available information, one can nevertheless state that the thermal parameters based on the insitu calibration with the Medtherm are the most reliable. Values based on material properties have a large degree of uncertainty, which adds support and weight to the insitu method of calibration adopted here.

\subsubsection{The interrogation area}

As mentioned before, an interrogation area of $15 \times 15$ pixels (corresponding to about $2.5 \mathrm{~mm} \times 2.5 \mathrm{~mm}$ ) had been used to compare TSP and Medtherm values - see Fig. 5. This size choice represents a trade-off between two counteracting aspects: the area must be large enough to deliver good statistical dynamics (especially at later times where the signals are quite low - see Fig. 5) on the one hand, but, on the other, small enough to avoid too much spatial smearing (the Medtherm has a $1.6 \mathrm{~mm}$ diameter, corresponding to an area of about $2.0 \mathrm{~mm}^{2}$ ). An analysis of the interrogation area for all 50 images led to a maximum value of relative error (based on the mean and standard deviation) of $2 \%$ for the intensities (Figs. 5 and 8), and for temperatures of $\pm 2 \mathrm{~K}$ at $292 \mathrm{~K}$ and $\pm 4 \mathrm{~K}$ at $340 \mathrm{~K}$ (see Fig. 10); similar uncertainties are to be expected for the heat loads. These error estimates are based solely on the statistics of counts in the interrogation region. They do not represent the total error/uncertainty - see later.

\subsubsection{Correction for time response of the paint}

This is potentially a larger source of uncertainty in the final heat load values, influencing both the time and amplitude axes. After excitation by LED light of the Eu complexes in the OV322 paint to an excited electronic state, these Eu molecules relax back to their ground state with a characteristic luminescence relaxation time $\tau$. Generally one assumes a mono-exponential decay (Liu and Sullivan 2005), so that $\tau$ represents just one simple decay process characterized by just this one constant. The decay process is temperaturedependent, with temperature influencing the rate of thermal quenching of the excited molecular state: use is in fact made of this phenomenon in the TSP lifetime method, where measurement of intensity decay rather than relative intensity is used by Ondrus et al. (2015). They measured $\tau$ as a function of temperature and pressure for the OV322 paint - see Fig. 15 .

Since pressures in the HEG run were around $100 \mathrm{kPa}$ (see Fig. 7 earlier), $\tau$ is then seen to be about $330 \mu$ s and $200 \mu$ s at $25^{\circ} \mathrm{C}$ and $50^{\circ} \mathrm{C}$, respectively. These are long times, of duration similar to that of the camera exposure time $\left(t_{E}=\right.$ $200 \mu \mathrm{s})$ in the HEG measurement with CW LED excitation. Hence, for any given exposure window (image), the measured signal is made up of components from fluorescence excited in this window plus decaying fluorescence excited in previous windows. This leads to a time smearing of the 


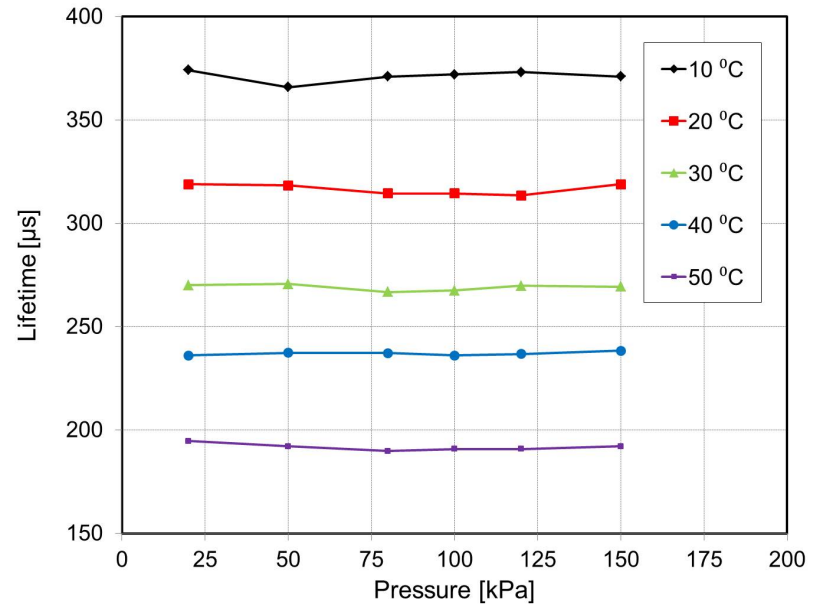

Fig. 15 Luminescence lifetimes $\tau$ of OV322 paint as a function of temperature and pressure $p$ (see text). (Source: Ondrus et al. (2015))

results over some windows, and perhaps also to an influence on the measured intensity in the window (especially so if the fluorescence intensity changes significantly from one window to the next). The following is an assessment of the influence of this effect on ensuing uncertainties in both time and amplitude, and will be carried out based on the following assumptions 3

1. Fluorescence decay time $\tau \approx 400 \mu \mathrm{s}\left(=2 t_{E}\right)$;

2. Decay over several windows is given by just the one decay constant;

3. Excitation is via a very short single LED pulse at the start of each window;

4. Only three contiguous windows are considered;

5. This analysis is considered to apply for a large number of single pulse excitations over the whole window (viz. simulating CW excitation);

6. Areas $A$ under the curves (see Fig. 16) are assumed to scale with initial intensities (see text).

The sketch of fluorescence decay curves in Fig. 16 (note: ordinates are not drawn to scale) show in the abscissa adjacent exposure time windows of width $t_{E}$ (where $t_{E}=200 \mu \mathrm{s}$ ). The signal measured by the camera in window $n$ (in green), is made up of the sum of contributions from excitation in windows $(n-2),(n-1)$ and $n$ itself, and will now be considered. For initial simplification, it is assumed that excitation in each of the windows $(n-2),(n-1)$ and $n$ occurs at the beginning (e.g. by a very short LED pulse), with ensuing fluorescence decay over three subsequent windows to the respective levels $1 / e^{3 / 2}, 1 / e^{1 / 2}$ and $1 / e$ of the initial fluorescence $I_{i}$. For example, fluorescence from window $(n-2)$ has

${ }^{3}$ It should be noted, that these assumptions were made in order to assess the measurement uncertainty for the described measurement case. It is not meant to be a general and rigorous analysis for all possible measurement conditions.

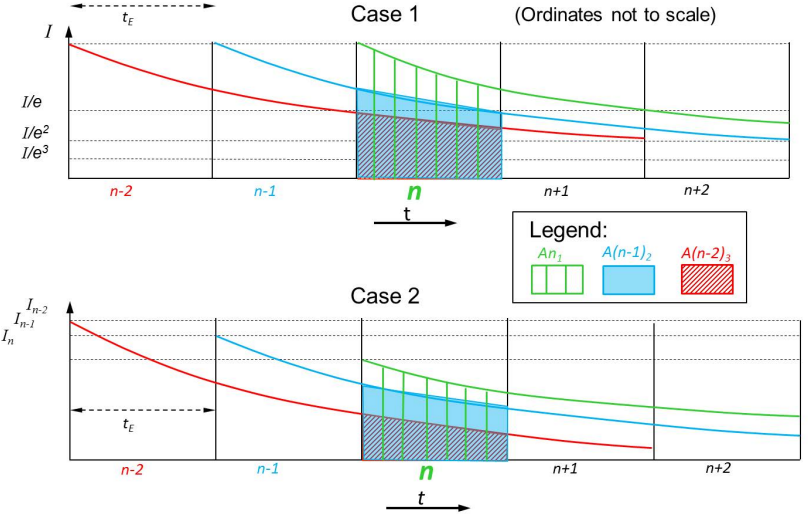

Fig. 16 Fluorescence decay curves over contiguous windows assuming single short pulse excitation at the beginning of each window. Constant (Case 1) and changing (Case 2) initial fluorescence intensities for the 3 windows are considered. (See text.) The areas $A$ pertain to those under the corresponding curves for fluorescence decay from windows $(n-2),(n-1)$ and $n$

fallen to a value of $1 / e^{3 / 2} \approx 22 \%$ of its original value at the end of window $n$; contributions from even earlier windows are not included in this assessment. Furthermore, for simplification, it has been assumed that $\tau \approx 2 t_{E}$ (this is approximately correct at the early times). The intensity measured by the camera in window $n$ is then $A(n-2)_{3}+A(n-1)_{2}+A n_{1}$ (see Fig. 16), namely:

$$
\begin{aligned}
I_{t o t} & =I_{n-2} \int_{2 t_{E}}^{3 t_{E}} e^{\left(-t / 2 t_{E}\right)} d t+I_{n-1} \int_{t_{E}}^{2 t_{E}} e^{\left(-t / 2 t_{E}\right)} d t \\
& +I_{n} \int_{0}^{t_{E}} e^{\left(-t / 2 t_{E}\right)} d t
\end{aligned}
$$

where $I_{n-2}, I_{n-1}$ and $I_{n}$ are the initial fluorescence intensities in their respective windows. For Case 1 in Fig. 16, it has been assumed that $I=I_{n-2}=I_{n-1}=I_{n}$, so that Eq. 4 simplifies to:

$$
\begin{aligned}
I_{\text {tot }} & =I\left[\int_{2 t_{E}}^{3 t_{E}} e^{\left(-t / 2 t_{E}\right)} d t+\int_{t_{E}}^{2 t_{E}} e^{\left(-t / 2 t_{E}\right)} d t+\int_{0}^{t_{E}} e^{\left(-t / 2 t_{E}\right)} d t\right] \\
& =I \int_{0}^{3 t_{E}} e^{\left(-t / 2 t_{E}\right)} d t
\end{aligned}
$$

Hence, from Fig. 16

$$
\begin{aligned}
& A n_{3}=I_{n-2}\left[\int_{2 t_{E}}^{3 t_{E}} e^{\left(-t / 2 t_{E}\right)} d t\right] \equiv A(n-2)_{3} \\
& A n_{3}=I_{n-2}\left[\int_{t_{E}}^{2 t_{E}} e^{\left(-t / 2 t_{E}\right)} d t\right] \equiv A(n-2)_{2}
\end{aligned}
$$

In other words, the measured signal would be the same as that which arises from capturing the fluorescence from just the one excitation at the start of window $\mathrm{n}$ for the three following windows $n, n+1$ and $n+2$. In Fig. 16 Case 1 this is shown pictorially: $A n_{1}+A n_{2}+A n_{3}=A n_{1}+A(n-1)_{2}+$ 
$A(n-2)_{3}$. Case 1 is the "trivial" case and applies only for equal initial fluorescence intensities. In Case 2 these intensities are not equal, but, for the measurements here, falling, as shown in Fig. 16 Case 2. (Obviously increasing temperature over time leads to decreasing fluorescence intensity.) Hence the contributions from windows $n-1$ and $n-2$ to intensity measured by the camera in window $n$ are larger, leading to a measured intensity which is too high: $A n_{3}<A(n-2)_{3}$ and $A n_{2}<A(n-1)_{2}$. The influence of this effect on the measurement uncertainty in window $\mathrm{n}$ will now be assessed, based on the measured differences between the intensities $I_{n-2}, I_{n-1}$ and $I_{n}$, as given by the results shown earlier in Fig. 5. here one sees that intensities drop by about $8 \%$ per window over the first three windows after gas arrival, where this drop is largest: $I_{n-2} / I_{n-1} \approx I_{n-1} / I_{n} \approx 0.92$. Assuming that the relative areas $A(n-2)_{3} / A n_{3}$ and $A(n-1)_{2} / A n_{2}$ (see Fig. 16 Case 2) scale with the initial intensities, namely:

$\frac{A(n-2)_{3}}{A n_{3}}=\frac{I_{n-2}}{I_{n}}=0.92^{2}$
$\frac{A(n-1)_{2}}{A n_{2}}=\frac{I_{n-2}}{I_{n}}=0.92$

the total intensity $I_{\text {tot }}$ measured in window $n$ then becomes:

$$
\begin{aligned}
I_{\text {tot }} & =A n_{1}+A(n-1)_{2}+A(n-2)_{3} \\
& =A n_{1}+A n_{2} \cdot \frac{I_{n-1}}{I_{n}}+A n_{3} \cdot \frac{I_{n-2}}{I_{n}} \\
& =A n_{1}+0.92 \cdot A n_{2}+0.92^{2} \cdot A n_{3}
\end{aligned}
$$

Now evaluate the integrals $A n_{1}, A n_{2}$ and $A n_{3}$ to get:

$A n_{1}: A n_{2}: A n_{3}=1: \frac{1}{e^{1 / 2}}: \frac{1}{e^{3 / 2}} \approx 100: 60: 36$

The discrepancies in the total signal $A n_{1}+A n_{2}+A n_{3}$ arise from the abovementioned differences for $A n_{2}$ vs. $A(n-1)_{2}$ and for $A n_{3}$ vs. $A(n-2)_{3}$, which correspond to, respectively, $(1-0.92)=0.08=8 \%$ and $(1-0.922)=0.15=15 \%$. This leads to a total signal of $100+60+36=196$, while the correction factor (or uncertainties) amount to $60 \cdot(-0.08)+$ $36 \cdot(-0.15)=-10$. The relative uncertainty is therefore about $-10 / 196=-0.05=-5 \%$. This is strictly not an error, but corresponds to a systematic discrepancy or a correction factor. Note that the sign is negative (since the intensity is dropping). The above discussion and estimations were based on an assumption of single short pulse LED excitation at the beginning of each window. However, CW LED excitation was used in the experiment. If one considers the $\mathrm{CW}$ excitation over a window to be made up of an infinite (viz. a large number) of single pulse excitations, then the conclusions reached here should still be valid, since each single pulse excitation at times within the window would behave and be evaluated in a similar way as above. The total uncertainty in amplitude (intensity), based only on those issues discussed in the preceding subsections, is hence estimated to be $2 \%$ and $-7 \%$. The uncertainty in the time domain, based on the model calculations discussed above, is $-2 t_{E}$, i.e. about $-0.4 \mathrm{~ms}$. These errors/uncertainties are included in the respective plots shown earlier - note they are asymmetrical.

\subsubsection{Normalized TSP and Medtherm temperatures}

Finally, a check on the performance of the TSP paint is to plot normalized temperature $T$ vs. time $t$, and assume that $T=f\left(t^{1 / 2}\right)$, or $T_{2}=f(t)-$ as suggested from Eq. 3 . If the behaviour of TSP (especially its time response) were to have a strong effect, one would expect the plots for TSP and Medtherm to show different trends over time. Fig. 17 shows a plot of $\left[\left(T_{t}-T_{0}\right) /\left(T_{\max }-T_{0}\right)\right]^{2}$ vs. $t$, where $T_{0}=$ temperature before gas arrival, $T_{\max }=$ temperature at $10 \mathrm{~ms}$ and $T_{t}=$ temperature at time $t$. Apart from the bumps in the Medtherm plot at early times, both plots show very similar behaviour, demonstrating that TSP, in spite of its larger time response (compared with Medtherm), can still deliver meaningful results of temperatures and thence of heat load.



Fig. 17 TSP and Medtherm temperatures T vs. time t, plotted normalized to minimum and maximum values, and assuming $T=f\left(t^{1 / 2}\right)$ (viz. $T_{2}=f(t)$ ): see Sect. 5.4.4 $T_{0}$ is the temperature before gas arrival; $T_{\max }$ is the temperature at $10 \mathrm{~ms}$. For error/uncertainty estimate: see Fig. 12

\section{Conclusions}

This work has demonstrated the usability of TSP to measure temperatures on a surface in a hypersonic, short-duration flow, and therefrom to calculate the heat load, making use of those assumptions generally applied to these types of flows. Both temperature and heat load results on the surface of a ramp model placed in a Mach $M=7.4$ HEG flow were shown. A Medtherm coaxial thermocouple situated at one 
position on the model was used to calibrate the TSP paint (viz. to obtain the thermal parameter $\rho c k$ for the paint). An average heat load in the interrogation region of $0.73 \pm 0.07 \mathrm{MWm}^{-2}$ has been measured. A further more detailed discussion of sources of error and uncertainty in the final results was given.

Acknowledgements The authors are grateful to the following for their assistance: Werner Sachs (DLR, now retired) for help in data processing using the DLR in-house code nToPas; Marco Costantini (DLR Göttingen) for invaluable contributions to determining the TSP paint material properties; Reinhard Sottong (DLR Cologne) for carrying out measurements of material properties on samples of paint; Jan Martinez Schramm (DLR Göttingen) for test preparation support; Vlado Ondrus (University of Hohenheim) for development of the paint OV322; Uwe Fey (DLR Göttingen) for construction of the LED multi-lens array.

\section{References}

Engineering ToolBox, 2015. engineeringtoolbox.com

URL WWW.

W. H. Beck, C. Klein, U. Henne, J. M. Schramm, A. Wagner, K. Hannemann, T. Gawehn, and A. Guelhan. Application of temperature and pressure sensitive paints to DLR hypersonic facilities: "lessons learned". In 53rd AIAA Aerospace Sciences Meeting, volume 2015-0023. American Institute of Aeronautics and Astronautics, 2015. doi $10.2514 / 6.2015-0023$.

W. J. Cook and E. J. Felderman. Reduction of data from thin-film heat-transfer gages - A concise numerical technique. AIAA Journal, 4(3):561-562, 1966. doi $10.2514 / 3.3486$.

M. Costantini, S. Risius, and C. Klein. Experimental investigation of the effect of forward-facing steps on boundary layer transition. Procedia IUTAM, 14:152-162, 2015. doi $10.1016 /$ j.piutam.2015.03.036

R. H. Engler, C. Klein, and O. Trinks. Pressure sensitive paint systems for pressure distribution measurements in wind tunnels and turbomachines. Measurement Science and Technology, 11(7):1077, 2000. doi:10.1088/09570233/11/7/320

U. Fey, C. Klein, V. Ondrus, S. Loose, and C. Wagner. Investigation of Reynolds number effects in high-speed train wind tunnel testing using temperature-sensitive paint. volume 44/2013, pages 29-47, Berlin, Germany, 2013. IFV Bahntechnik e.V.

A. D. Gardner, C. Klein, W. E. Sachs, U. Henne, H. Mai, and $\mathrm{K}$. Richter. Investigation of three-dimensional dynamic stall on an airfoil using fast-response pressure-sensitive paint. Experiments in Fluids, 55(9):1807, Sept. 2014. doi $10.1007 / \mathrm{s} 00348-014-1807-4$
J. W. Gregory, H. Sakaue, T. Liu, and J. P. Sullivan. Fast pressure-sensitive paint for flow and acoustic diagnostics. Annual Review of Fluid Mechanics, 46(1):303-330, Jan. 2014. doi 10.1146/annurev-fluid-010313-141304.

K. Hannemann and J. Martinez Schramm. High enthalpy, high pressure short duration testing of hypersonic flows. In C. Tropea, J. Foss, and A. Yarin, editors, Springer Handbook of Experimental Fluid Mechanics, pages 1081-1125. Springer-Verlag Berlin Heidelberg, 2007.

K. Hannemann, J. Martinez Schramm, and S. Karl. Recent extensions to the High Enthalpy Shock Tunnel Göttingen (HEG). In 2nd International ARA Days, Arcachon, Frankreich, 2008.

C. Klein, R. H. Engler, U. Henne, and W. E. Sachs. Application of pressure-sensitive paint for determination of the pressure field and calculation of the forces and moments of models in a wind tunnel. Experiments in Fluids, 39(2): 475-483, Aug. 2005. doi:10.1007/s00348-005-1010-8.

C. Klein, U. Henne, W. Sachs, S. Hock, N. Falk, U. Beifuss, V. Ondrus, and S. Schaber. Pressure Measurement on Rotating Propeller Blades by means of the PressureSensitive Paint Lifetime Method. In 51st AIAA Aerospace Sciences Meeting Including the New Horizons Forum and Aerospace Exposition, volume 2013-0483, Grapevine, Texas, USA, 2013. AIAA. doi $10.2514 / 6.2013-483$

C. Klein, U. Henne, W. Sachs, U. Beifuss, V. Ondrus, M. Bruse, R. Lesjak, and M. Löhr. Application of Carbon Nanotubes (CNT) and Temperature-Sensitive Paint (TSP) for the Detection of Boundary Layer Transition. In 52nd AIAA Aerospace Sciences Meeting, 2014-1482, Gaylord National, Maryland, USA, 2014.

S. Laurence, H. Ozawa, D. Lieber, J. Martinez Schramm, and K. Hannemann. Investigation of Unsteady/QuasiSteady Scramjet Behavior using High-Speed Visualization Techniques. In 18th AIAA/3AF International Space Planes and Hypersonic Systems and Technologies Conference, Tours, France, Sept. 2012. American Institute of Aeronautics and Astronautics. doi 10.2514/6.2012-5913.

S. J. Laurence, A. Wagner, and K. Hannemann. Schlierenbased techniques for investigating instability development and transition in a hypersonic boundary layer. Experiments in Fluids, 55(8):1782, Aug. 2014. doi:10.1007/s00348-014-1782-9.

T. Liu and J. P. Sullivan. Pressure and Temperature Sensitive Paints. Experimental Fluid Mechanics. Springer-Verlag Berlin Heidelberg, 2005.

J. Martinez Schramm, K. Hannemann, H. Ozawa, W. Beck, and C. Klein. Development of temperature sensitive paints in the High Enthalpy Shock Tunnel Göttingen, HEG. Lisbon, Portugal, Mar. 2015.

T. Nagayama, H. Nagai, H. Tanno, and T. Komuro. Global heat flux measurement using temperature-sensitive paint 
in High-Enthalpy Shock Tunnel HIEST. In 55th AIAA Aerospace Sciences Meeting, AIAA SciTech Forum. American Institute of Aeronautics and Astronautics, Jan. 2017. doi $10.2514 / 6.2017-1682$.

K. Nakakita, T. Osafune, and K. Asai. Global heat transfer measurement in a hypersonic shock tunnel using temperature-sensitive paint. In 41st Aerospace Sciences Meeting and Exhibit, Aerospace Sciences Meetings. American Institute of Aeronautics and Astronautics, Jan. 2003. doi $10.2514 / 6.2003-743$.

S. Ohmi, H. Nagai, K. Asai, and K. Nakakita. Effect of TSP layer thickness on global heat transfer measurement in hypersonic flow. In 44th AIAA Aerospace Sciences Meeting and Exhibit, Aerospace Sciences Meetings. American Institute of Aeronautics and Astronautics, Jan. 2006. doi 10.2514/6.2006-1048

V. Ondrus, R. J. Meier, C. Klein, U. Henne, M. Schäferling, and U. Beifuss. Europium 1,3-di(thienyl)propane-1,3diones with outstanding properties for temperature sensing. Sensors and Actuators A: Physical, 233:434-441, Sept. 2015. doi:10.1016/j.sna.2015.07.023.

H. Ozawa, S. J. Laurence, J. M. Schramm, A. Wagner, and K. Hannemann. Fast-response temperaturesensitive-paint measurements on a hypersonic transition cone. Experiments in Fluids, 56(1):1853, Jan. 2015. doi 10.1007/s00348-014-1853-y.

D. Peng, L. Jiao, Z. Sun, Y. Gu, and Y. Liu. Simultaneous PSP and TSP measurements of transient flow in a longduration hypersonic tunnel. Experiments in Fluids, 57 (12):188, Dec. 2016. doi 10.1007/s00348-016-2280-z.

D. L. Schultz and T. V. Jones. Heat-transfer measurements in short-duration hypersonic facilities. Technical Report AGARD-AG-165, Advidsory Group for Aerospace Research and Development, Paris, France, Feb. 1973.

T. Streit, K. Horstmann, G. Schrauf, S. Hein, U. Fey, Y. Egami, J. Perraud, I. S. El Din, U. Cella, and J. Quest. Complementary numerical and experimental data analysis of the ETW Telfona pathfinder wing transition tests. In 49th AIAA Aerospace Sciences Meeting Including the New Horizons Forum and Aerospace Exposition. American Institute of Aeronautics and Astronautics, Jan. 2011. doi 10.2514/6.2011-881.

A. Wagner, M. Kuhn, J. M. Schramm, and K. Hannemann. Experiments on passive hypersonic boundary layer control using ultrasonically absorptive carbon-carbon material with random microstructure. Experiments in Fluids, 54(10):1606, Oct. 2013. doi $10.1007 / \mathrm{s} 00348-013-1606-$ 3 . 